\title{
O CUIDADO À PESSOA COM DIABETES MELLITUS E SUA FAMÍLIA
}

Soraia Schoeller Dornelles ${ }^{1}$, Denise Maria Guerreiro Vieira da Silva², Mariza Maria Serafim Mattosinho, Adriana Eich Kuhen ${ }^{4}$, Soraya Baião ${ }^{5}$, Rita Sandoval ${ }^{6}$

\begin{abstract}
RESUMO: Estudo exploratório descritivo, com abordagem qualitativa, teve como objetivos compreender as práticas de pessoas com diabetes mellitus no seu convívio domiciliar. Participaram 23 pessoas, diagnosticadas pela equipe de saúde com risco de glicemia instável e atendidas pela enfermagem do ambulatório do Hospital Universitário. Foram realizadas entrevistas semiestruturadas e observação sistemática, entre 2010 e 2011, cujos dados foram analisados através da análise temática. Emergiram dos dados duas categorias: adesão ao tratamento e apoio da família. O estudo evidenciou a importância do enfermeiro em cuidar da família e não só da pessoa com diabetes mellitus, orientando-a e compartilhando seus conhecimentos para que essa tenha melhor qualidade de vida. DESCRITORES: Cuidados de enfermagem; Visita domiciliar; Diabetes mellitus.
\end{abstract}

\section{THE CARE FOR THE PERSON WITH DIABETES MELLITUS AND THEIR FAMILY}

ABSTRACT: This descriptive-exploratory study with a qualitative approach aims to understand the practices of people with diabetes mellitus in the co-existence at home. 23 people participated, diagnosed by the health team as being at risk of unstable glycemia, and attended by the nursing staff of the Outpatient Department of a University Hospital. Semi-structured interviews were held and systematic observation made, between 2010 and 2011, the data being analyzed by means of thematic analysis. Two categories emerged from the data: adherence to the treatment and family support. The study evidenced the importance of the nurse in caring for the family, and not just the person with diabetes mellitus, guiding her and sharing knowledge such that she may have better quality of life. DESCRIPTORS: Nursing care; Home visits; Diabetes mellitus.

\section{EL CUIDADO A LA PERSONA CON DIABETES MELLITUS Y SU FAMILIA}

RESUMEN: Estudio exploratorio descriptivo, con abordaje cualitativo que tuvo como objetivo comprender las prácticas de personas con diabetes mellitus en su dinámica domiciliar. Participaron 23 personas, diagnosticadas por el equipo de salud con riesgo de glucemia inestable y atendidas por la enfermería del ambulatorio del Hospital Universitario. Fueron realizadas entrevistas semiestructuradas y observación sistemática, entre 2010 y 2011, cuyos datos fueron analizados por medio del análisis temático. De los datos, resultaron dos categorías: Adhesión al tratamiento y Apoyo de la familia. El estudio evidenció la importancia del enfermero en cuidar de la familia y no solamente de la persona con diabetes mellitus, orientándola y compartiendo sus conocimientos para que esta tenga más calidad de vida.

DESCRIPTORES: Cuidados de enfermería; Visita en domicilio; Diabetes mellitus.

\footnotetext{
${ }^{1}$ Enfermeira. Doutora em Filosofia da Saúde e Enfermagem. Professora do Departamento de Enfermagem da Universidade Federal de Santa Catarina - UFSC. Membro do Núcleo de Estudos e Assistência em Enfermagem e Saúde a Pessoas em Condição Crônica NUCRON.

${ }^{2}$ Enfermeira. Doutora em Enfermagem. Professora do Departamento de Enfermagem e do Programa de Pós-Graduação em Enfermagem - PEN UFSC. Coordenadora do NUCRON. Bolsista de produtividade do CNPq.

${ }^{3}$ Enfermeira da Secretaria do Estado da Saúde de Santa Catarina. Mestre em Enfermagem.

${ }^{4}$ Enfermeira. Especialista em Enfermagem Cardiovascular. Mestranda pelo PEN UFSC. Bolsista CAPES REUNI. Membro do Grupo de Estudos da História do Conhecimento da Enfermagem e Saúde - GEHCES.

${ }^{5}$ Enfermeira do Hospital Universitário da UFSC. Mestranda pelo Mestrado Profissional PEN UFSC.

${ }^{6}$ Enfermeira do Hospital Universitário da UFSC. Mestre em Enfermagem.
}

Autor correspondente:

Recebido: 06/08/2012

Soraia Schoeller Dornelles

Aprovado: 30/06/2013

Universidade Federal de Santa Catarina

Rua Prof. M ${ }^{\mathrm{a}}$ Flora Pausewang, s/n - 88036-800 - Florianópolis-SC-Brasil

E-mail: soraia.dornelles@ufsc.br

Cogitare Enferm. 2013 Jul/Set; 18(3):496-501 


\section{INTRODUÇÃO}

Em situações crônicas de saúde, como é o caso do Diabetes mellitus (DM), a necessidade dos cuidados relativos ao tratamento, controle da doença, dificuldades sociais e familiares encontradas, são de difícil aceitação para a pessoa e sua família. Elas terão que se adaptar a várias mudanças na sua maneira de viver exigidas pela nova condição de saúde, o que pode levar a uma desorganização da estrutura familiar, na qual todos devem contribuir no cuidado, orientando, estimulando e controlando novos hábitos ${ }^{(1)}$.

No cuidado de uma pessoa em condição crônica é preciso ir além do conhecimento sobre suas alterações físicas e psíquicas, cabendo à enfermagem, e demais profissionais da saúde, reconhecer estes e outros aspectos, compartilhar os conhecimentos e traçar coletivamente (profissional/pessoa/sua família) caminhos para um viver mais saudável ${ }^{(2)}$.

A educação em saúde é essencial no cuidado às pessoas com DM, ao mesmo tempo em que é elemento crítico deste processo, pois requer o envolvimento efetivo entre os profissionais, as pessoas com o agravo e seus familiares. É importante que essa educação considere para além da patologia em questão, mas também a história de vida, cultura, conhecimento sobre a doença e habilidades individuais e familiares para lidarem com o problema ${ }^{(3)}$.

Tal patologia implica em compromisso constante com hábitos saudáveis de vida e compreensão do seu problema de saúde para cuidar-se. A educação em saúde, focada somente na transmissão de conhecimentos e ensino de habilidades para o autocuidado, não tem apresentado um efeito prático ${ }^{(4)}$. No cuidado verticalizado, o profissional é detentor do saber e a pessoa com DM tratada como desprovida deste conhecimento. É preciso mudar esta prática, considerando os saberes do profissional e da pessoa, dialogando, vendo a pessoa em seu contexto e, a partir daí, construindo juntos, profissional e pessoa com DM, estratégias no modo de se cuidar, que é único e singular para cada pessoa e família.

Tal prática encontra respaldo na Constituição Brasileira de 1988 e nas políticas públicas daí advindas, que transformaram o modelo de atenção à saúde, inserindo os princípios de universalidade, equidade e participação social. Como parte integrante desta nova lógica foi implantado o Programa de Saúde da Família e institucionalizada a visita domiciliária realizada pelos trabalhadores em saúde do referido programa $^{(5)}$. Com esta visita, busca-se, entre outras coisas, o conhecimento das condições concretas de vida da pessoa e sua família, para detectar problemas intervenientes no autocuidado ${ }^{(6)}$. A realidade dos domicílios é, possivelmente, diferente daquela percebida pelos relatos apresentados na consulta de enfermagem no ambulatório.

O objetivo deste estudo foi compreender as práticas de pessoas com DM no convívio domiciliar, a intenção foi identificar as dificuldades destas pessoas em manterem seus níveis glicêmicos aceitáveis, a partir das orientações dadas nas consultas de enfermagem. As orientações diziam respeito à alimentação, armazenagem de insulina e cuidados na sua aplicação, bem como, orientações sobre a realização de atividades físicas associadas ao bem estar físico.

\section{MÉTODO}

Trata-se de um estudo exploratório com abordagem qualitativa. A seleção dos participantes ocorreu no ambulatório de um Hospital de Ensino da Grande Florianópolis-Santa Catarina. A amostra foi intencional e constituída por 23 pessoas portadoras de DM. Durante as entrevistas elas estavam sempre acompanhadas por um familiar de maior idade, os entrevistados tinham idade entre 18 e 86 anos, com DM tipo 1 ou 2, atendidas, ao menos, em três vezes em consultas de enfermagem, e que continuassem, após estas três consultas, com glicemia instável e/ou acima dos padrões preconizados.

Com estas pessoas foi agendado uma visita domiciliar para ampliar o processo educativo. Previamente à realização da visita domiciliar essas pessoas foram convidadas a integrar o estudo. Aquelas que concordaram, foram entrevistadas utilizando um roteiro semiestruturado com questões sobre hábitos alimentares, atividade física, armazenamento da medicação, higiene corporal, ações realizadas pelo cuidador e pela pessoa. Os familiares que estavam presentes também foram convidados a participar, também abordando o cotidiano de cuidados. O momento da visita também foi utilizado para a observação sobre as ações de cuidado ao DM.

As entrevistas e observações foram registradas no próprio roteiro, tomando algumas notas durante a entrevista e complementando as informações o mais brevemente possível, ou seja, no mesmo dia em que foram realizadas. Cada visita durou em média três horas, sendo que as entrevistas duraram cerca de 40 minutos. Durante a visita, além da entrevista, foram 
observados questões de saneamento básico, condições de moradia, armazenamento de medicações, auto aplicação da insulina (nos pacientes que faziam uso), local de armazenamento da insulina, hábitos alimentares, todos os aspectos importantes para o cuidado de um portador de DM, aspectos sócio-econômicos-culturais. Os dados foram coletados no período de julho de 2010 a julho de 2011. O período da coleta se deu devido à forma da escolha da população estudada.

Foram seguidos os princípios éticos previstos na Resolução n. 196/96 (7), e o projeto foi aprovado por Comitê de Ética sob número 827 em 28 de junho de 2010. Os participantes do estudo foram esclarecidos sobre o tema da pesquisa, seus objetivos, e de que a sua participação era voluntária, não havendo danos pessoais ou qualquer tipo de encargo financeiro, além de não interferir de forma alguma com o atendimento realizado no ambulatório e na visita domiciliar.

A análise dos dados se deu com sua organização e consolidação do conjunto de informações de forma a favorecer a emersão dos temas ${ }^{(8)}$. Inicialmente, cada entrevista e observação realizada foram analisadas isoladamente com a leitura e releitura sucessiva das entrevistas e das observações, seguida da codificação das falas e dos registros das observações. Os códigos foram reunidos por semelhança de modo que expressassem as ações de cuidados realizados pelas pessoas com DM e seus familiares resultando em duas categorias que passam a ser apresentadas a seguir: Adesão ao tratamento e Apoio da familia.

\section{RESULTADOS}

Cabe salientar ainda que as pessoas entrevistadas com DM, ao discorrerem sobre suas práticas de cuidado, tiveram algumas destas falas contrastantes com a observação das pesquisadoras, evidenciando que nem sempre o que as pessoas expressam reflete sua prática ou é percebido da mesma forma pelos profissionais da saúde.

Dentre as 23 pessoas que integraram o estudo, 13 eram do sexo feminino e 10 do sexo masculino e 20 concluiram o ensino fundamental.

\section{Adesão ao tratamento}

Este estudo nos possibilitou observar que as pessoas com DM efetuaram algumas mudanças em suas vidas, após terem recebido orientações dos enfermeiros nas consultas de enfermagem, acerca de sua condição crônica e dos cuidados e tratamentos como diminui- ção da utilização de doces e carboidratos, cuidado com os pés, e manuseio da insulina e sua utilização. Por outro lado, também foi observado nos domicilios que algumas ações acordadas durante as consultas de enfermagem não eram seguidas da forma como os profissionais haviam indicado, como, por exemplo, os cuidados com a alimentação. Mesmo com uma afirmação inicial de que seguiam a dieta indicada, ao serem questionados sobre qual havia sido a alimentação do dia anterior, listavam pão branco com geléia no café da manhã, arroz, feijão, batata e carne de panela no almoço, pão no lanche da tarde, arroz, feijão e macarrão no jantar. Quanto à observação nestes domicílios foi possível verificar que dispunham de grande variedade de alimentos ricos em carbohidratos, tais como pães, pizzas, massas, refrigerantes não diets ou lights, dentre outros. Poucas vezes havia frutas e ou verduras e, mesmo quando questionados sobre a existência desses alimentos, invariavelmente, a resposta era que não dispunham desse tipo de alimento, ora por serem muito caros, ora por não gostarem.

Com relação à atividade física, a quase totalidade dessas pessoas referiram não praticar qualquer tipo de atividade, mesmo aquelas que já apresentavam algum tipo de complicação pelo DM. Atividade física não era referido como integrando as mudanças necessárias para cuidar do DM. Apesar de serem pessoas que já estavam em tratamento há algum tempo e tendo recebido orientações sistemáticas acerca de sua condição crônicas, percebemos que as mesmas tinham dificuldades de compreensão sobre sua condição de saúde e os cuidados diários. Isso se expressou, por exemplo, no uso da insulina, com acondicionamento inapropriado, grande número de frascos vencidos há mais de 60 dias; realização de rodízio inadequado na sua aplicação, e desconhecimento sobre os riscos do não controle da glicemia capilar.

\section{Apoio da família}

A família teve papel de destaque, apoiando as pessoas com DM no processo saúde e doença. Todos os familiares denotaram preocupação e mostravam-se disponíveis e interessados nos cuidados, especialmente com relação à dieta.

No entanto, isso não significava que contribuíam, efetivamente, nos cuidados, pois faziam interpretações acerca do que lhes era orientado de forma diferente da intenção das orientações efetuadas pela enfermagem. Uma esposa, ao ser orientada sobre a importância de 
inserir peixe na alimentação do marido, passou a fazer peixe frito e peixe com pirão dois dias na semana. A intenção era sempre de querer ajudar seus familiares, porém, essa ajuda nem sempre era convergente ao controle indicado pelos profissionais.

Houve casos em que sentimentos de proteção, pena, companheirismo sobrepuseram-se ao do controle mais rigoroso da alimentação, e visavam compensar as limitações impostas pela doença como exemplificado no depoimento a seguir:

[...] aqui em casa a gente come pizza uma vez por semana, e a gente sempre traz um docinho para ela [...] ela gosta, e de vez em quando, também não faz tão mal assim [...] se não, nem adianta viver, né? A vida já é tão amarga [...]. M.S.S.

Por outro lado, também apreendemos que a família é aliada no cuidado à pessoa com $\mathrm{DM}$, evidenciando-se como o principal apoio que possuem, constituindo o cenário na qual acontecem as avaliações e as tomadas de decisões sobre o modo de cuidar, como os extratos da fala:

[...] depois que a gente descobriu que ele tinha diabetes, todos passaram a fazer dieta aqui em casa, se não, não adianta [...] ele acaba comendo o que a gente come [...] então, passamos a comer mais vezes por dia, 5 ou 6 vezes, e todo o dia tem salada, pão integral [...] tiramos os doces, o café agora é com adoçante e passamos a comer mais frutas. C.P.S.

\section{DISCUSSÃO}

Para que o cuidado seja realizado adequadamente, os familiares e a pessoa com DM precisam compreender o que é a doença e quais implicações da realização, ou não, dos cuidados e porque devem ser realizados ${ }^{(9)}$.

A educação em saúde tem papel essencial para a mudança de comportamento, cabendo ao enfermeiro, e demais profissionais envolvidos, reconhecerem este processo, compartilhando conhecimentos que ambos têm sobre sua condição, traçando caminhos para uma viver mais saudável ${ }^{(10)}$. O cuidado é fundamental para a manutenção da vida e as pessoas envolvidas neste processo desenvolvem, gradativamente, a compreensão da sua realidade e dos modos de cuidar. Assim, as pessoas com DM buscam uma vida com mais qualidade, que pode ser construída e consolidada num processo que inclui a reflexão sobre o que é decisivo para viver com sua condição de saúde com mais qualidade, superando as dificuldades encontradas ${ }^{(11)}$.

Cuidar no domicílio é uma ação prestada ao indivíduo e família com a finalidade de promover conforto, alívio e qualidade de vida, restabelecendo e promovendo o autocuidado. O desenvolvimento do cuidado domiciliar envolve conhecimento, responsabilidade, habilidades técnicas, pedagógicas e psicológicas na qual o enfermeiro deve ser um aliado, um parceiro da família e da pessoa com $\mathrm{DM}^{(12)}$.

Há ações de cuidado para as quais as pessoas com DM deste estudo e seus familiares não têm o mesmo entendimento dos profissionais, seja simplesmente não aderindo, seja interpretando de maneira diversa as orientações das consultas de enfermagem, como o caso do armazenamento da insulina ou da alimentação. No caso da insulina, não se pode afirmar que as pessoas não consideraram o pactuado na consulta de enfermagem, pois a mesma estava guardada em ambiente limpo, em local específico para isso, porém não no local indicado (na parte inferior da geladeira). Em estudo de reflexão sobre a aderência, foi destacada a importância de conhecer o porque as pessoas não aderem, sendo que aspectos sociais, especialmente os relacionamentos, têm especial influência ${ }^{(13)}$.

Nem sempre a família cuidava da pessoa com DM na mesma perspectiva dos profissionais da saúde, o que já foi observado por outros autores em suas pesquisas ${ }^{(10-11)}$. De certa forma, isso prejudica o controle da doença, porém, não indica que a família não esteja preocupada ou não apoie. $\mathrm{O}$ apoio é observado em diferentes momentos e inclui a forma como a família prepara as refeições, estimula a participação nas atividades físicas, no uso correto das medicações e no controle das glicemias ${ }^{(3,11)}$.

Ressalta-se que a família influencia nas relações estabelecidas entre a pessoa com diabetes e os profissionais de saúde referente às informações dadas. Ela tem suas próprias tradições, valores, estabelecendo seus próprios padrões para interação dentro e fora do convívio familiar, tornando complexa a ação educativa. Mudar comportamentos requer que os profissionais tenham conhecimentos sobre o contexto social, econômico, religioso e cultural destas pessoas para sensibilizá-las para um cuidado domiciliar positivo, proporcionando melhor controle da doença, com consequência na qualidade de vida de ambos.

O diabetes é uma doença crônica que irá acompanhar a pessoa para o resto de sua vida fazendo com que ela passe a ter certas limitações e cuidados ${ }^{(14)}$. Assim, podemos dizer que o tempo poderá ser um aliado, ou não, da equipe de saúde para um cuidado efetivo. 
Aliado, quando possibilita planejar com tranquilidade as ações necessárias a curto, médio e longo prazo, analisando de que forma a pessoa com diabetes e sua família as executam, permitindo caminharem juntas construindo alternativas de cuidado e enfrentamento. Negativamente, no sentido de que as complicações podem ir se estabelecendo e a qualidade de vida sendo comprometida, caso os cuidados sejam adiados.

Ficou evidente que maior aproximação entre a família e a equipe de saúde é essencial para ambos, possibilitando uma assistência direcionada às necessidades dessas pessoas, resultando em uma orientação pertinente à realidade ${ }^{(11)}$.

\section{CONCLUSÃO}

Este estudo objetivou conhecer quais as ações de cuidados as pessoas realizam a partir do que foi orientado nas consultas de enfermagem, bem como o apoio dado pela família.

Os resultados mostram que o cuidado nem sempre é convergente ao que os profissionais de saúde esperam. Ressalta-se, nesse processo, a importante participação dos familiares, que promovem o cuidado indicado pelos profissionais, interpretando como estes devem ser feitos. Nesse sentido, o que fica mais evidente é a necessidade de inclusão, mais efetiva, dos familiares no processo educativo.

Prestar cuidado no domicílio consiste em uma abordagem de cuidar e reabilitar a pessoa em seu contexto familiar. No entanto, saber considerar as diferentes classes sociais e econômicas, respeitando a privacidade, valores e crenças de cada uma delas, torna-se um desafio para os profissionais de saúde.

Os achados desse estudo indicam que a assistência à saúde a pessoas com DM requer profissionais comprometidos, que possuam conhecimentos, habilidades e atitudes para perceber as várias especificidades que o cuidado requer. Inclui o reconhecimento de que há um conjunto único e indivisível: pessoas com DM, contexto domiciliar, família, cuidador, equipe multiprofissional, rede social e as relações entre os mesmos. Nesse sentido, o planejamento, a organização e a coordenação das ações de cuidado precisam atender às demandas específicas de cada pessoa.

\section{REFERÊNCIAS}

1. Mattosinho MMS, Silva DM. Itinerário terapêutico do adolescente com diabetes mellitus tipo 1 e seus familiares. Rev. Latino-Am Enfermagem. 2007;15(6):1113-9.

2. Hermann AP, Lacerda MR. Atendimento Domiciliar a Saúde: um Relato de Experiência. Cogitare enferm. 2007;12(4):513-8.

3. Funnell MM, Brown TL, Childs BP, Haas LB, Hosey GM, Jensen B, et al. National Standards for Diabetes Self-Management Education. DIABETES CARE. [Internet] 2008;33(suppl. 1):89-96.

4. Grossi SAA, Pascali PM. Cuidados de Enfermagem em Diabetes Mellitus: Manual de Enfermagem. Sociedade Brasileira de Diabetes, São Paulo [Internet] 2009 [acesso em 3 mar 2010]. Disponível: http://www.diabetes.org. br/attachments/1118_1324_manual_enfermagem.pdf.

5. Ministério da Saúde (BR). Secretária de Atenção a Saúde. Núcleo Técnico da Política Nacional de Humanização. Acolhimento nas práticas de produção da Saúde - Ministério da Saúde, Secretária de Atenção a Saúde, Núcleo Técnico da Política Nacional de Humanização. $2^{\mathrm{a}}$ ed. Brasilia: Editora do Mistério da Saúde; 2010.

6. Delamater AM, Improving Patient Adherence. Clinical Diabetes. 2006:24(2):71-7.

7. Brasil. Conselho Nacional de Saúde. Resolução n. 196/96. Diretrizes e Normas Regulamentadoras de Pesquisas envolvendo Seres Humanos. [Internet] 2010 [acesso em 10 mar 2010] Disponível: http://conselho. saude.gov.br/docs/Resolucoes/Reso196.doc.

8. Minayo MCS, organizadora. Pesquisa Social: Teoria, Método e Criatividade. 22a ed. RJ: Vozes; 2003.

9. Francioni FF, Silva DM. O processo de aceitação do viver com diabetes mellitus: considerações sobre a influência do meio ambiente. Texto Contexto Enferm. 2002;11(3):36-43.

10. Silva I, Pais-Ribeiro J, Cardoso H. Adesão ao tratamento da Diabetes Mellitus: A importância das características demográficas e clínicas. Revista Referência. 2006;2(2):33-41.

11. Silva MD. Narrativas do viver com diabetes mellitus:experiencias pessoais e culturais. Florianopolis: PEN/UFSC; 2001.

12. Baggio SC, Mazzola JC, Marcon SS. A vivência da pessoa com diabetes após atendimento de urgência. Cogitare enferm. 2011;16(2):275-81 
13. Souza SS, Bonetti A, Meirelles BHS, Mattosinho MMS, Coelho MS, Argenta C. Viver com doença crônica em uma comunidade pesqueira. Acta Paul. Enferm. 2010;23(2):194-9.

14. Sakata KN, Almeida MCP, Alvarenga AM, Craco $\mathrm{PF}$, Pereira MJB. Concepções da equipe de saúde da família sobre as visitas domiciliares. Rev. bras. enferm. 2007;60(6): 659-64. 